\title{
AFP-producing hepatoid adenocarcinoma (HAC) of peritoneum and omentum: a case report and literature review
}

This article was published in the following Dove Press journal: OncoTargets and Therapy

\author{
Man Zou ${ }^{1, *}$ \\ Yanhui $\mathrm{Li}^{2}, *$ \\ Yuhong Dai' \\ Li Sun ${ }^{1}$ \\ Tingting Huang' \\ Xianglin Yuan' \\ Hong Qiu' \\ 'Department of Oncology, Tongji \\ Hospital, Tongji Medical College, \\ Huazhong University of Science and \\ Technology, Wuhan 430030, People's \\ Republic of China; ${ }^{2}$ Department of \\ Cardiology, Tongji Hospital, Tongji \\ Medical College, Huazhong University of \\ Science and Technology, Wuhan 430030, \\ People's Republic of China
}

*These authors contributed equally to this work
Correspondence: Hong Qiu

Department of Oncology, Tongji Hospital, Tongji Medical College, Huazhong University of Science and Technology, Wuhan 430030, People's Republic of China

Tel/Fax +862783662836

Email tjqiuhong@163.com

\begin{abstract}
Hepatoid adenocarcinoma (HAC) is a group of neoplasms with features resembling hepatocellular carcinoma. The stomach is the most commonly affected organ among the reported primary sites. We report the case of a 28 -year-old man with chronic hepatitis B and a complaint of abdominal distension. The patient was examined by PET-CT and magnetic resonance imaging (MRI), which showed diffuse thickening of the peritoneum and omentum but no mass was found in the liver. Pathological examination of a biopsy of the omental nodules was consistent with moderately differentiated hepatocellular carcinoma (HCC); thus, a diagnosis of HAC of the peritoneum and omentum was established. The patient received a chemotherapy regimen consisting of oxaliplatin and capecitabine and gained remarkable effects as the AFP level dropped significantly, and the tumour nearly disappeared. When the patient shifted to the standard multikinase inhibitors, Sorafenib or Lenvatinib, both treatments were ineffective. HAC is a heterogeneous group of prognostically unfavourable tumours mimicking the histological appearance of $\mathrm{HCC}$, and the treatment outcomes are still unclear.
\end{abstract}

Keywords: AFP producing, peritoneal hepatoid carcinoma, XELOX chemotherapy, multikinase inhibitors

\section{Introduction}

Hepatoid adenocarcinoma (HAC) is a rare primary extrahepatic malignant neoplasm that shares a similar morphology and immunohistotechemistry to hepatocellular carcinoma (HCC). It has been reported to occur in the stomach, oesophagus, pancreas, ovary, lung, colon, gallbladder, uterus and other sites. ${ }^{1-8}$ The stomach is the most commonly affected primary organ among these sites. Peritoneal hepatoid carcinoma (PHC) is extremely rare, to the best of our knowledge, with only 7 cases reported to date in the English literature. ${ }^{9}$ Among these cases, only 2 presented with diffuse peritoneal nodules. ${ }^{10,11}$ Herein, we report a case of AFP-producing HAC of the peritoneum and omentum and discuss its clinical, radiological and histopathological features as well as its treatment and prognosis in combination with related literature.

\section{Case presentation}

A 28-year-old male patient with a previous history of HBV mother-to-child transmission presented with a complaint of abdominal distension for half a month and no 
other associated symptoms. The physical examination revealed no pathological findings other than shifting dullness positive. A diagnostic abdominal puncture was performed. The cytology of ascites showed abundant blood cells, a small amount of lymphocytes, mesothelial cells and nothing else. PET-CT indicated thickening of the peritoneum and omentum which is hypermetabolic with an SUVmax of 2.5 (Figure 1A). Abdominal MRI revealed that peritoneal and omental thickening with nodules, suggestive of malignant disease (Figures 1B and 2A). There was no remarkable abnormality in the liver.

Laboratory tests showed that the complete blood count and liver function were almost within the normal range (except for albumin $30.8 \mathrm{~g} / \mathrm{L}$ ). Hepatitis B virus surface antigen (HBs $\mathrm{Ag}$ ) was seropositive, and hepatitis $\mathrm{B}$ virus DNA was $1.45^{*} 10^{\wedge} 3 \mathrm{IU} / \mathrm{mL}$. Serum alpha-fetoprotein (AFP) was dramatically elevated $(>60,500 \mathrm{ng} / \mathrm{mL})$. Serum of carbohydrate antigen 125 (CA-125) was slightly elevated (CA-125 1343.6 U/mL) The remaining tumour markers, including $\beta-\mathrm{HCG}$, were within the normal range.

Exploratory laparotomy was carried out for the purposes of diagnosis and treatment. Intraoperatively, after aspirating of $4000 \mathrm{~mL}$ bloody ascites, densely packed nodules approximately the sizes of green peas were observed from the left diaphragm, right diaphragm, omentum and peritoneum following the surface of the pelvic walls. The liver has a smooth surface without granular nodules. Since it is difficult to perform a complete excision and removed a tumour intact, a biopsy of the omental nodules was performed. Pathological examination of a biopsy of the omental nodules was consistent with moderately differentiated HCC. The neoplastic cells were immunoreactive for Glypican 3, Arginase I, $\alpha$-fetoprotein (AFP), CK8/18, and CD34, weakly positive for PCK, CK19, and CK7 and negative for EMA, hepatocytes, CK20, calretinin, and VIM (Figure 3A-F).

As the tumour was surgically unresectable, after treatment with liver protection drugs and nutrition support, the patient underwent a cytotoxic chemotherapy regimen consisting of oxaliplatin and capecitabine every 3 weeks for 7 cycles. During this period of review, AFP declined sharply from $60,500 \mathrm{ng} / \mathrm{mL}$ to $450 \mathrm{ng} / \mathrm{mL}$ and with the complete regression of ascites (Figure 4). After 2 cycles, MRI revealed that all the pre-existing lesions were disappeared (Figure 2B). Four cycles and 6 cycles later, MRI revealed a significant constant clinical response. However, due to liver function damage in which serum bilirubin was increased, the patient received only capecitabine monotherapy in the 8 th chemotherapy cycle. Then the review revealed recurrence in the greater omentum and under the liver capsule.

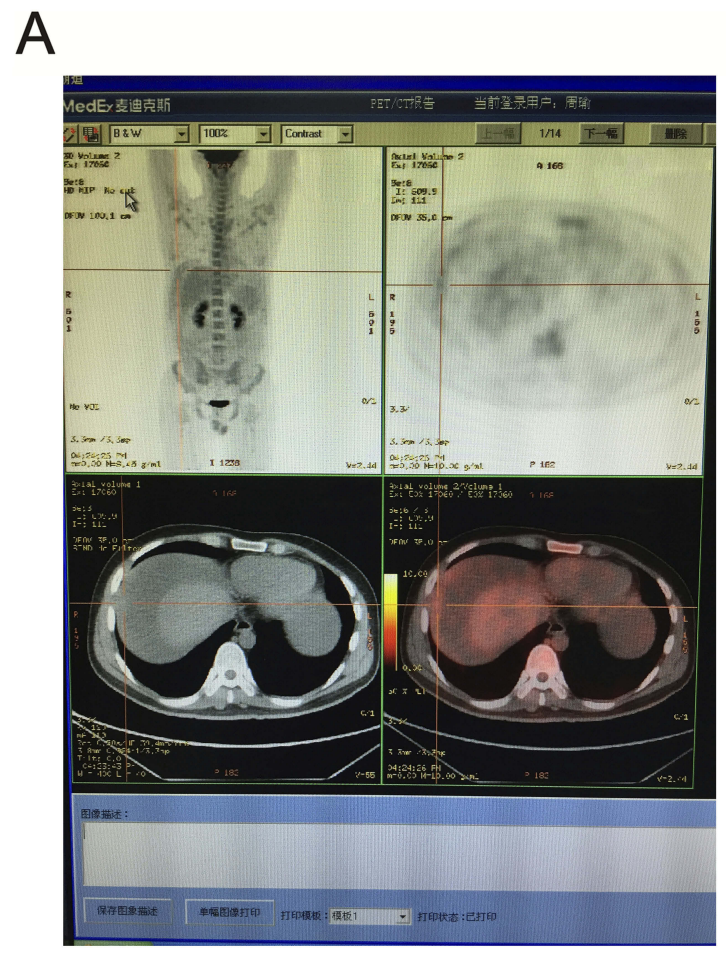

B
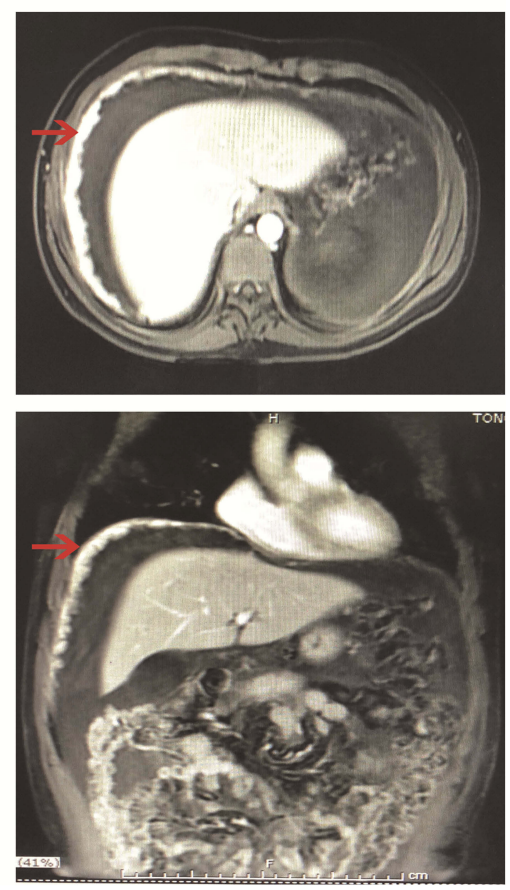

Figure I PET-CT and MRI results showed multiple masses located in the peritoneum and omentum. 
A
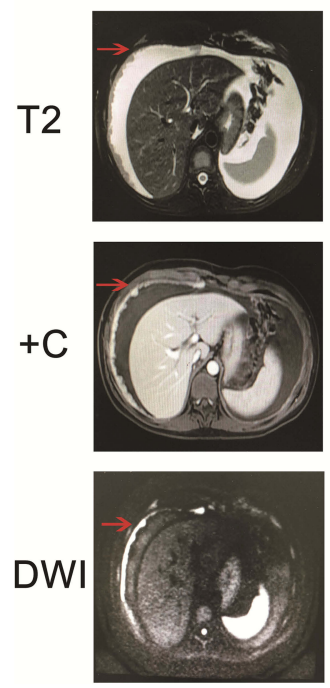

B
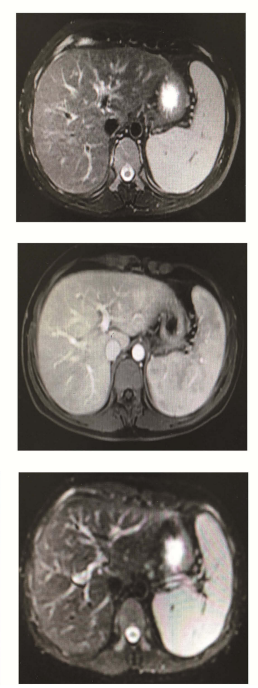

C
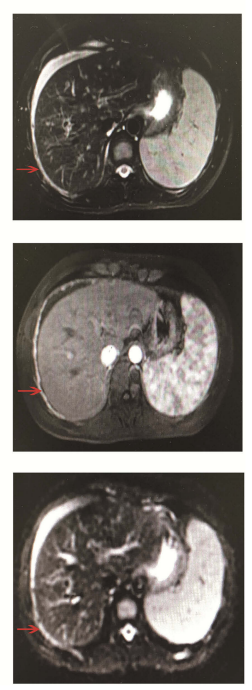

Figure 2 MRI revealed tumour masses at different stages of the disease including (A) pretreatment (B) post-treatment and (C) recurrence.

Later, the patient was given the multikinase inhibitor Sorafenib at the dose of $400 \mathrm{mg}$ twice a day for one month. The obvious side effects were hypertension, second-grade hand-foot syndrome and a decreased platelet count. MRI revealed stabilization of the disease and new sub-capsular hydrops of the liver, while the serum AFP level increased again to $22,312 \mathrm{ng} / \mathrm{mL}$ (Figure 2C). At the time, the liver function of the patient returned to a normal level. He again underwent XELOX chemotherapy for 2 cycles. During the treatment, there was an obvious decline in AFP (once again to the lowest point of $7900 \mathrm{ng} / \mathrm{mL}$ ). The patient did not come for further treatment; therefore, a MRI review was not performed. He took Lenvatinib for treatment for one month at a dose of $12 \mathrm{mg}$ per day. Although his subjective symptoms, including hypertension and hand-foot syndrome, were more manageable than those on Sorafenib, the platelet count still decreased accompanied by an increase in the AFP level to $31,261 \mathrm{ng} / \mathrm{mL}$. MRI revealed stable disease compared to review after the use of Sorafenib. He then began to undergo the previous chemotherapy regimen for 4 cycles. After 2 cycles, his AFP level dropped to $10,546 \mathrm{ng} / \mathrm{mL}$. At the time, MRI revealed shrinkage of the lesions. However, after 4 cycles, the AFP level increased to $45,007 \mathrm{ng} / \mathrm{mL}$, and MRI showed that the disease progressed, with the repeated emergence of ascitic fluid. The patient became resistant to XELOX chemotherapy and refused to take other chemotherapy regimens. As a result, we recommended that he undergo the whole-genome sequencing and also identify the predicators of immunotherapy. Because the specimen was limited, peripheral blood was used to perform whole-genome sequencing as
A

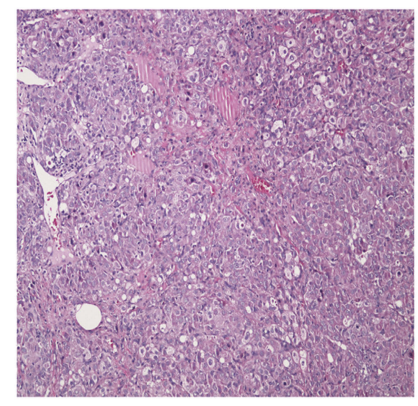

D

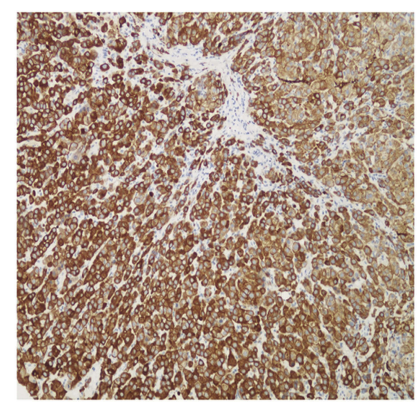

B

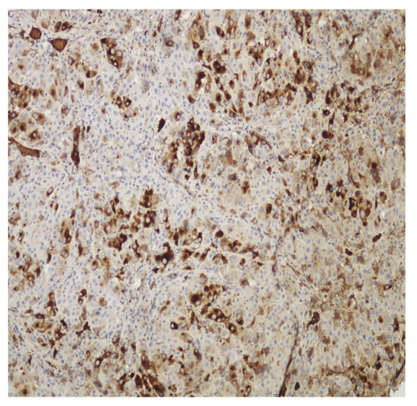

$\mathrm{E}$

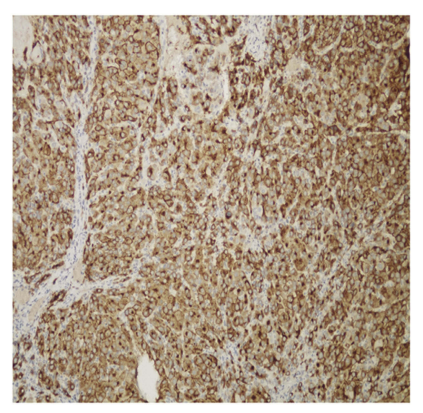

C

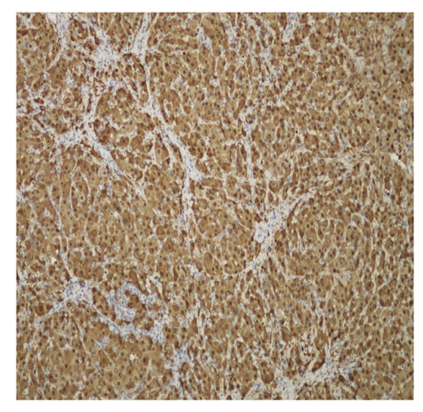

$\mathrm{F}$

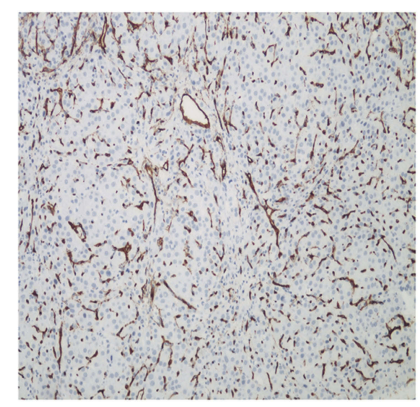

Figure 3 (A) Hepatocellular carcinoma composed of cells exhibited an eosinophilic cytoplasm and enlarged and round nuclei (haematoxylin \& eosin, stain 200) (B) The tumour cells were positive for $\boldsymbol{\alpha}$-fetoprotein (AFP) (AFP stain I00) (C) The tumour cells were positive for Arginase I (Arginase I stain I00) (D) The tumour cells were positive for CK8/I 8 (CK8/18 stain 100) (E) The tumour cells were positive for Glypican 3 (Glypican 3 stain 100) (F) The tumour cells were positive for CD34 (CD34 stain I00). 


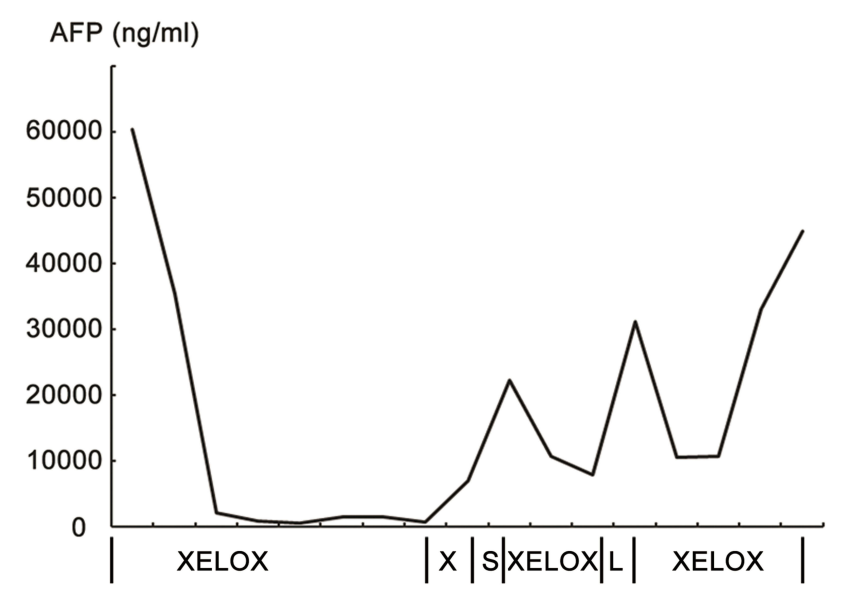

Figure 4 The chart shows the general trend of serum AFP at different stages of the treatment.

Abbreviations: XELOX, Oxaliplatin and Capecitabine, X: Capecitabine; S, Sorafenib; L, Lenvatinib.

well as the PD-L1 expression test and the tumour mutational burden (TMB) test. The result turned out there was no recommended new targeted therapy for the patient; PD-L1 expression was less than $1 \%$; and TMB was 58 mut/Mb. Therefore, we recommended PD-L1 inhibitor immunotherapy in this patient. He then began immunotherapy in another hospital. The results, which were conveyed via telephone follow-up, confirmed that the ascitic fluid of the patient continue to increase; therefore, an abdominal cavity drainage tube was placed to relieve the symptoms of abdominal distension while receiving the immunotherapy. During the treatment period, serum AFP was not tested, but routine blood tests and blood biochemical examinations showed no obvious abnormality. To date, the survival time of this patient is 16 months, with an ECOG performance status of 0 and a Child-Pugh score of A.

\section{Discussion}

HAC is defined as a primary extrahepatic carcinoma with morphological resemblance to HCC. ${ }^{12}$ Due to its rarity and variable presentation, treatment is far from being standardized and its diagnosis was mainly based on the pathology and exclusion of primary liver lesions.

AFP, as a biomarker, is elevated in testicular cancer and liver cancer patients. ${ }^{13}$ In our case, since MRI showed no evidence of a lesion in the liver, testicular cancer was suspected. Ultrasonography and PET-CT were performed to exclude metastases from other primary sites. However, both ultrasonography and PET-CT revealed a normal testis, and the patient's $\beta$-HCG concentration was within the normal limits. These findings were helpful to definitively exclude a germ cell tumour which was the most likely possible alternative to HAC not only due to the high level of $\beta$-HCG, but also due to the young age of this patient.

Since diffuse or multiple tumours are localized in the peritoneal cavity, tuberculous peritonitis may also be considered. High levels of adenosine deaminase (ADA) are used for diagnosing tuberculosis. ${ }^{14}$ In the present case, ADA level in ascites was very low (5 IU/L). As PET-CT revealed no obvious tumour in other organs and, above all, the final pathological report excluded primary peritoneal mesothelioma, a diagnosis of HAC in the peritoneum was thus established.

Although immunohistochemical stains have limited sensitivity and specificity, the staining differences confirm and highlight gross and routine microscopic features. Based on the morphology and staining pattern and correlations with intraoperative findings, the nodules represent a hepatoid tumour.

In a literature review performed by Metzgeroth et al, the median age at diagnosis of HAC was 65 years (range 21-88 years).HAC occurs more frequently in elderly men with a male to female ratio of $2.4: 1$, except in gallbladder HAC and gynaecological tumours. The clinical symptoms of HAC may vary depending on the anatomic location of the tumour. $^{15}$ Among the reported more than 200 cases of HAC, other than peritoneal HAC, most tumours first undergo surgical treatment to remove the primary lesion. In some cases, adjuvant chemotherapy is performed to reduce the high risk of reccurence. ${ }^{15}$ In addition, peritoneal HAC can be divided into two subtypes: one presents as large masses, and the other presents as diffuse nodules. ${ }^{16}$ Usually, patients with localized tumours undergo excision or debulk operation followed by chemotherapy. The prognosis of these patients is better than that of those who are not able to undergo an operation. As Dr Lucas reported which is in accordance with us, after a woman diagnosed as HAC of the peritoneal cavity receives debulking surgery, 5-fluorouracil, leucovorin and oxaliplatin (FOLFOX) chemotherapy prevented the recurrence of disease for more than 3 years. ${ }^{16}$ Both studies reported diffuse HAC in the peritoneum, and in the case we covered here, the primary tumour was unresectable, so palliative systemic treatment was performed. Treatment involved chemotherapy and Sorafenib targeted therapy. The mean survival time of patients with diffuse peritoneal HAC is only 6 months, which is less than the mean survival time of resectable HAC from other primary sites. ${ }^{16}$ In the present case, the XELOX regimen was performed, which is different from the paclitaxel plus 
cisplatin regime in most other cases. Our decision was based on a study on HCC that showed modest activity and a durable response with the use of fluorouracil combined with oxaliplatin. ${ }^{17}$ Only a few HACs of the colon and rectum have been reported to use a chemotherapy regimen of oxaliplatin combined with fluorouracil and resulted in a mean survival time of 19.3 months, the longest survival time of all cases. ${ }^{18}$

What is particularly interesting about our case is that the patient was insensitive to the standard first-line therapy drugs such as Sorafenib or Lenvatinib but sensitive to the XELOX chemotherapy regimen. ${ }^{19,20}$ Our finding is consistent with those of Pandey, in which HAC of the ovary showed no response to Sorafenib. ${ }^{4}$ This suggests that despite the pathologic resemblance between HAC and HCC, they are biologically different. As treatment began, the level of AFP dramatically decreased, and the tumour disappeared. When one chemotherapy drug was removed due to the side effect of liver damage, the AFP level increased accordingly. When we reused the same combined chemotherapy drugs, tumour markers markedly declined. Our patient had an excellent response to chemotherapy treatment and showed very minor side effects. Metzgeroth et al reported that more than $90 \%$ of HAC cases present with increased serum AFP. ${ }^{15}$ Based on our findings and those from others, the AFP serum level is useful in the upcoming follow-up of AFP-producing hepatoid cancer.

For advanced and unresectable patients, under the precondition of effective treatment, reduced side effects of chemotherapy and improved tolerance will offer more advantages to patients. Considering the patient's desire to not continue undergoing cytotoxic chemotherapy, as a subsequent treatment, we can take a chance on newly proposed regimens. Regorafenib and Nivolumab are the only FDA-approved second-line treatments for HCC after progression on Sorafenib or chemotherapy. ${ }^{21,22}$ In consideration of the past ineffective usage of multikinase inhibitor drugs, we then implemented immunotherapy recommended by NCCN guidelines. Several predictors predetermine immunotherapy efficacy, including PD-L1 expression, the MMR status and TMB. Although PD-L1 expression was low and there were not enough tumour specimens to detect the MMR status, the TMB was 58 mut/ $\mathrm{Mb}$, which provides an opportunity for immunotherapy. It is challenging to obtain adequate tumour tissue for molecular testing, but it has been reported that a blood-based assay to measure TMB in plasma (bTMB) is a clinically actionable biomarker for predicting immunotherapy efficacy in NSCLC, with a cutoff of $16 .^{23}$ Hence, our patient was a proper candidate for immunotherapy, and we hope that this patient will benefit with a better quality of life.

\section{Conclusion}

In summary, $\mathrm{HAC}$ is a heterogeneous group of prognostically unfavourable tumours mimicking the histological appearance of HCC. However, data are limited, there is no standard treatment and outcomes are still unclear. From this case report, XELOX chemotherapy could be a suitable treatment choice for primary peritoneal HAC. We are still investigating the management and prognosis of this rare tumour and we will continually follow-up on subsequent treatments.

\section{Abbreviations}

HAC, hepatoid adenocarcinoma; HCC, hepatocellular carcinoma; PHC, Peritoneal hepatoid carcinoma; HBs Ag, Hepatitis B virus surface antigen; AFP, $\alpha$-fetoprotein; TMB, tumour mutational burden; ADA, adenosine deaminase.

\section{Ethics approval and consent to participate}

The patient provided written informed consent for the case details and images to be published and this study was approved by the Ethics Committee of Tongji Hospital of Huazhong University of Science and Technology.

\section{Acknowledgment}

This work was supported by National Natural Science Foundation of China (No.81700348) for modifying the language and publishing.

\section{Disclosure}

The authors report no conflicts of interest in this work.

\section{References}

1. Liu X, Cheng Y, Sheng W, et al. Analysis of clinicopathologic features and prognostic factors in hepatoid adenocarcinoma of the stomach. Am J Surg Pathol. 2010;34(10):1465-1471. doi:10.1097/PAS.0b013e3181f0a873

2. Chiba N, Yoshioka T, Sakayori M, et al. AFP-producing hepatoid adenocarcinoma in association with Barrett's esophagus with multiple liver metastasis responding to paclitaxel/CDDP: a case report. Anticancer Res. 2005;25(4):2965-2968.

3. Kuo PC, Chen SC, Shyr YM, et al. Hepatoid carcinoma of the pancreas. World J Surg Oncol. 2015;13:185. doi:10.1186/s12957-015-0586-6

4. Pandey M, Truica C. Hepatoid carcinoma of the ovary. J Clin Oncol. 2011;29(15):e446-e448. doi:10.1200/JCO.2010.33.6321

5. Basse V, Schick U, Gueguen P, et al. A mismatch repair-deficient hepatoid adenocarcinoma of the lung responding to anti-PD-L1 durvalumab therapy despite no PD-L1 expression. J Thorac Oncol. 2018;13 (7):e120-e122. doi:10.1016/j.jtho.2018.03.004 
6. Armaghani A, Hernandez Gonzalo D, Daily K. Hepatoid adenocarcinoma of the colon. BMJ Case Rep. 2015;2015:bcr2014206222. doi:10.1136/bcr-2014-206222

7. Karayiannakis AJ, Kakolyris S, Giatromanolaki A, et al. Hepatoid adenocarcinoma of the gallbladder: case report and literature review. $J$ Gastrointest Cancer. 2012;43(Suppl 1):S139-S144. doi:10.1007/ s12029-011-9326-5

8. Kato K, Suzuka K, Osaki T, et al. Primary hepatoid adenocarcinoma of the uterine cervix. Int J Gynecol Cancer. 2007;17(5):1150-1154. doi:10.1111/j.1525-1438.2007.00901.x

9. Oyan B, Sonmez O, Eren OO, et al. Primary peritoneal hepatoid adenocarcinoma: response to platin based chemotherapy. $J$ Clin Oncol. 2016;34(suppl; abstr e15667):e15667-e15667. doi:10.1200/ JCO.2016.34.15_suppl.e15667

10. Kitamura H, Ikeda K, Honda T, et al. Diffuse hepatoid adenocarcinoma in the peritoneal cavity. Intern Med. 2006;45(19):1087-1091. doi:10.2169/internalmedicine. 45.1760

11. Gopaldas R, Kunasani R, Plymyer MR, et al. Hepatoid malignancy of unknown origin-a diagnostic conundrum: review of literature and case report of collision with adenocarcinoma. Surg Oncol. 2005;14 (1):11-25. doi:10.1016/j.suronc.2004.09.011

12. Kishimoto T, Nagai Y, Kato K, et al. Hepatoid adenocarcinoma: a new clinicopathological entity and the hypotheses on carcinogenesis. Med Electron Microsc. 2000;33(2):57-63. doi:10.1007/s007950070002

13. Liu P, Li W, Song HC, et al. Characteristics, treatment decisions and outcomes of prepubertal testicular germ cell tumour: a descriptive analysis from a large Chinese center. J Pediatr Urol. 2018;14(5):443. e1-443.e7. doi:10.1016/j.jpurol.2018.02.030

14. Riquelme A, Calvo M, Salech F, et al. Value of adenosine deaminase (ADA) in ascitic fluid for the diagnosis of tuberculous peritonitis: a meta-analysis. J Clin Gastroenterol. 2006;40(8):705-710. doi:10.1097/ 00004836-200609000-00009

15. Metzgeroth G, Strobel P, Baumbusch T, et al. Hepatoid adenocarcinoma - review of the literature illustrated by a rare case originating in the peritoneal cavity. Onkologie. 2010;33(5):263-269. doi:10.1159/ 000305717
16. Lucas ZD, Shah M, Trivedi A, et al. Hepatoid adenocarcinoma of the peritoneal cavity: prolonged survival after debulking surgery and 5fluorouracil, leucovorin and oxaliplatin (FOLFOX) therapy. $J$ Gastrointest Oncol. 2012;3(2):139-142.

17. Qin S, Bai Y, Lim HY, et al. Randomized, multicenter, open-label study of oxaliplatin plus fluorouracil/leucovorin versus doxorubicin as palliative chemotherapy in patients with advanced hepatocellular carcinoma from Asia. J Clin Oncol. 2013;31(28):3501-3508. doi:10.1200/JCO.2012.44.5643

18. Chen Y, Schaeffer DF, Yoshida EM. Hepatoid adenocarcinoma of the colon in a patient with inflammatory bowel disease. World $J$ Gastroenterol. 2014;20(35):12657-12661. doi:10.3748/wjg.v20.i35. 12657

19. Llovet JM, Ricci S, Mazzaferro V, et al. Sorafenib in advanced hepatocellular carcinoma. $N$ Engl J Med. 2008;359(4):378-390. doi:10.1056/NEJMoa0708857

20. Kudo M, Finn RS, Qin S, et al. Lenvatinib versus sorafenib in firstline treatment of patients with unresectable hepatocellular carcinoma: a randomised phase 3 non-inferiority trial. Lancet. 2018;391 (10126):1163-1173. doi:10.1016/S0140-6736(18)30207-1

21. Bruix J, Qin S, Merle P, et al. Regorafenib for patients with hepatocellular carcinoma who progressed on sorafenib treatment (RESORCE): a randomised, double-blind, placebo-controlled, phase 3 trial. Lancet. 2017;389(10064):56-66. doi:10.1016/S0140-6736(16) 32453-9

22. El-Khoueiry AB, Sangro B, Yau T, et al. Nivolumab in patients with advanced hepatocellular carcinoma (CheckMate 040): an open-label, non-comparative, phase $1 / 2$ dose escalation and expansion trial. Lancet. 2017;389(10088):2492-2502. doi:10.1016/S0140-6736(17) 31046-2

23. Gandara DR, Paul SM, Kowanetz M, et al. Blood-based tumor mutational burden as a predictor of clinical benefit in non-small-cell lung cancer patients treated with atezolizumab. Nat Med. 2018;24 (9):1441-1448. doi:10.1038/s41591-018-0134-3
OncoTargets and Therapy

\section{Publish your work in this journal}

OncoTargets and Therapy is an international, peer-reviewed, open access journal focusing on the pathological basis of all cancers, potential targets for therapy and treatment protocols employed to improve the management of cancer patients. The journal also focuses on the impact of management programs and new therapeutic agents and protocols on patient perspectives such as quality of life, adherence and satisfaction. The manuscript management system is completely online and includes a very quick and fair peer-review system, which is all easy to use. Visit http://www.dovepress.com/ testimonials.php to read real quotes from published authors. 